\title{
Recent advances and future prospects in choroideremia
}

\author{
This article was published in the following Dove Press journal: \\ Clinical Ophthalmology \\ 23 November 2015 \\ Number of times this article has been viewed
}

\author{
Martin S Zinkernagel 1,2 \\ Robert E MacLaren ${ }^{3,4}$ \\ 'Department of Ophthalmology, \\ ${ }^{2}$ Department of Clinical Research, \\ Inselspital, Bern University Hospital, \\ and University of Bern, Bern, \\ Switzerland; ${ }^{3}$ Nuffield Laboratory \\ of Ophthalmology, University of \\ Oxford and Oxford Eye Hospital, \\ Oxford University NHS Trust \\ NIHR Biomedical Research Centre, \\ Oxford, UK; ' ${ }^{4}$ Moorfields Eye Hospital \\ NIHR Biomedical Research Centre, \\ London, UK
}

\begin{abstract}
Choroideremia is a complex and rare disease that is frequently misdiagnosed due to its similar appearance to classic retinitis pigmentosa. Recent advances in genetic testing have identified specific genetic mutations in many retinal dystrophies, and the identification of the mutation of the CHM gene on the $\mathrm{X}$ chromosome 25 years ago has paved the way for gene replacement therapy with the first human trials now underway. This article reviews the epidemiological and pathological features of choroideremia and new prospects in imaging to monitor disease progression, as well as potential treatment approaches for choroideremia.
\end{abstract}

Keywords: choroideremia, gene replacement therapy, retinal imaging, clinical trials

\section{Introduction}

Choroideremia is an X-linked, recessive disease of the retina resulting in progressive degeneration of the retina, the retinal pigment epithelium (RPE), and the choroid. The prevalence of choroideremia is estimated to be approximately 1:50,000 in people of European descent. ${ }^{1}$

The disease is characterized by the clinical appearance of atrophy of the choroid resulting in a characteristic pale color of the fundus, originating from the translucence of the white sclera (Figure 1). In males, the condition gradually advances and starts with night blindness and eventually results in progressive loss of peripheral vision and total blindness in the late stages, although some extreme peripheral field detection may remain. ${ }^{2}$ However, disease progression is relatively slow and the fovea is generally only affected in the end stages of the disease. Therefore, patients may retain good central visual function as late as 50-70 years old. ${ }^{3,4}$

Although X-linked diseases preferentially occur in men (as they have only one $\mathrm{X}$ chromosome) choroideremia may also present in female carriers. Carrier females may show characteristic pigmentary changes of the fundus with patchy chorioretinal degeneration. This is due to the process of lyonization in which one copy of the $\mathrm{X}$ chromosome is silenced ${ }^{5}$ and therefore cells carrying the mutation are intermixed with cells expressing the normal $\mathrm{X}$ chromosome resulting in a mosaic pattern of the disease. Therefore, most female carriers are asymptomatic and maintain normal visual acuity. The characteristic fundus appearance and a family history consistent with X-linked disease are sufficient for the diagnosis of choroideremia. Other retinal dystrophies with similar fundus appearance can usually be ruled out by family history or laboratory testing. These include autosomal recessive gyrate atrophy which can usually be ruled out by family history and testing for hyperornithinemia, Oliver-McFarlane syndrome, a PNPLA6-related disorder and dominantly negative
Correspondence: Martin S Zinkernagel Department of Ophthalmology, Inselspital, Bern University Hospital, and University of Bern, Freiburgstrasse, 3010 Bern, Switzerland

Email m.zinkernagel@gmail.com 

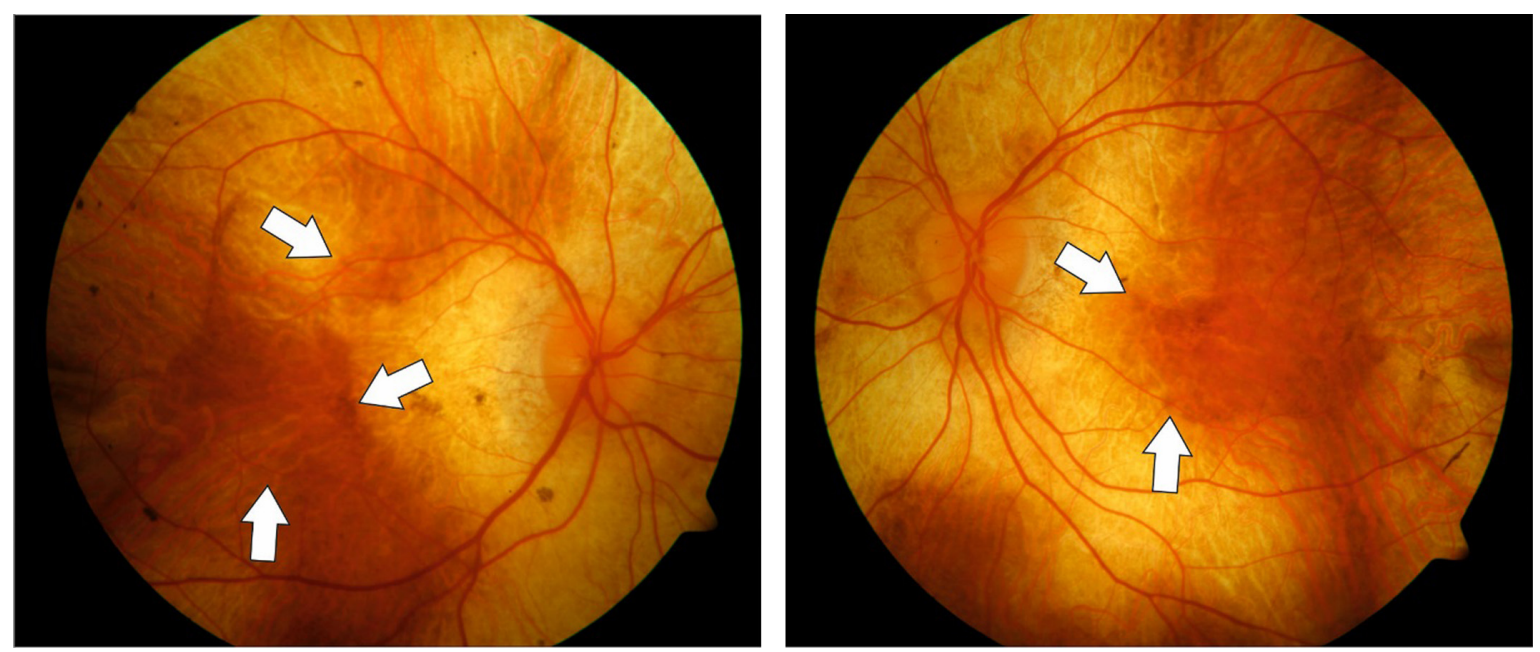

Figure I Fundus color photographs of the right and left eye of a patient with advanced choroideremia. Note: There is a residual island of healthy tissue in the central macula and fovea (white arrows).

mutations in RPE65 or bifocal chorioretinal atrophy, and an autosomal dominant disease with a gene mutation on chromosome $6 .^{6-8}$

There are several reports on the histological changes in choroideremia - this is critically important for designing treatments, because it is essential to know which retinal cells are affected by the $C H M$ gene deficiency and therefore need to be targeted by gene therapy vectors. In postmortem histological sections, independent degeneration of choriocapillaris, RPE, and neurosensory retina have been described. Furthermore, areas of relatively well-preserved retinal architecture were found adjacent to areas of severe degeneration, corresponding to the clinical fundus appearance., ${ }^{9,10}$ Photoreceptor degeneration was less pronounced above preserved RPE, but occasionally photoreceptor loss was seen in areas with preserved RPE. ${ }^{11}$ Within the affected areas, the retina was thinned due to thinning of outer segments of the photoreceptors and loss of nuclei within the outer nuclear layer. Subsequently, loss of nuclei within the inner nuclear layer was found as well. ${ }^{11}$ In addition, there was profound rarefication of most vasculature and melanocytes within the choroid. There is some evidence that inflammation occurs within the retina in patients with choroideremia: a recent report has provided evidence of a mild T-lymphocytic infiltration within the choroid. ${ }^{11}$ Furthermore, pigment filled macrophages have been identified in the subretinal space associated with rosette formation of the retina. ${ }^{12}$ Whereas inflammation is not likely to play an active part in the pathogenesis of choroideremia, there seems to be a reactive inflammatory response in the areas of active disease as photoreceptor debris usually is phagocytized and removed by macrophages. ${ }^{13}$

\section{Retinal imaging in choroideremia and implications for gene replacement therapy}

In the last decade, advances in retinal imaging have led to an improved understanding of morphological changes occurring during the disease course of choroideremia. Optical coherence tomography (OCT) can be used to diagnose macular changes associated with choroideremia such as choroidal neovascularization, ${ }^{14}$ cystoid macular edema,${ }^{15}$ epiretinal membrane formation, ${ }^{16}$ outer retinal tubulations, ${ }^{17}$ macular hole formation, ${ }^{18}$ and macular hole complicated by retinal detachment. ${ }^{19}$ Fundus autofluorescence shows a characteristic speckled pattern of low- and high-density fundus autofluorescence (Figure 2) ${ }^{20}$ Increased lipofuscin accumulation originating from digestion of degenerating rods by RPE cells is thought to cause increased autofluorescence in choroideremia, whereas low density fluorescence reflects loss of the RPE layer whilst the choroid is still intact. This is corroborated by functional tests, showing an association of multifocal electroretinogram (mfERG) deterioration with major fundus autofluorescence changes. ${ }^{20,21}$ Despite high resolution OCT and genetic characterization of choroideremia, the sequence of morphological changes leading to vision loss remains unknown. There are several theories on which retinal layers are primarily affected by choroideremia. Whereas some reports show that the RPE is the first layer to degenerate, followed by loss of photoreceptors, ${ }^{22}$ others claim that the photoreceptor layer, ${ }^{23}$ in particular the rods, ${ }^{24}$ is primarily affected, followed by degeneration of the RPE and the choriocapillaris. The latter hypothesis is not however supported by the observations from patients with dominant RPE65 mutations who have a very similar choroidal atrophy appearance to $\mathrm{CHM}$. Since the 


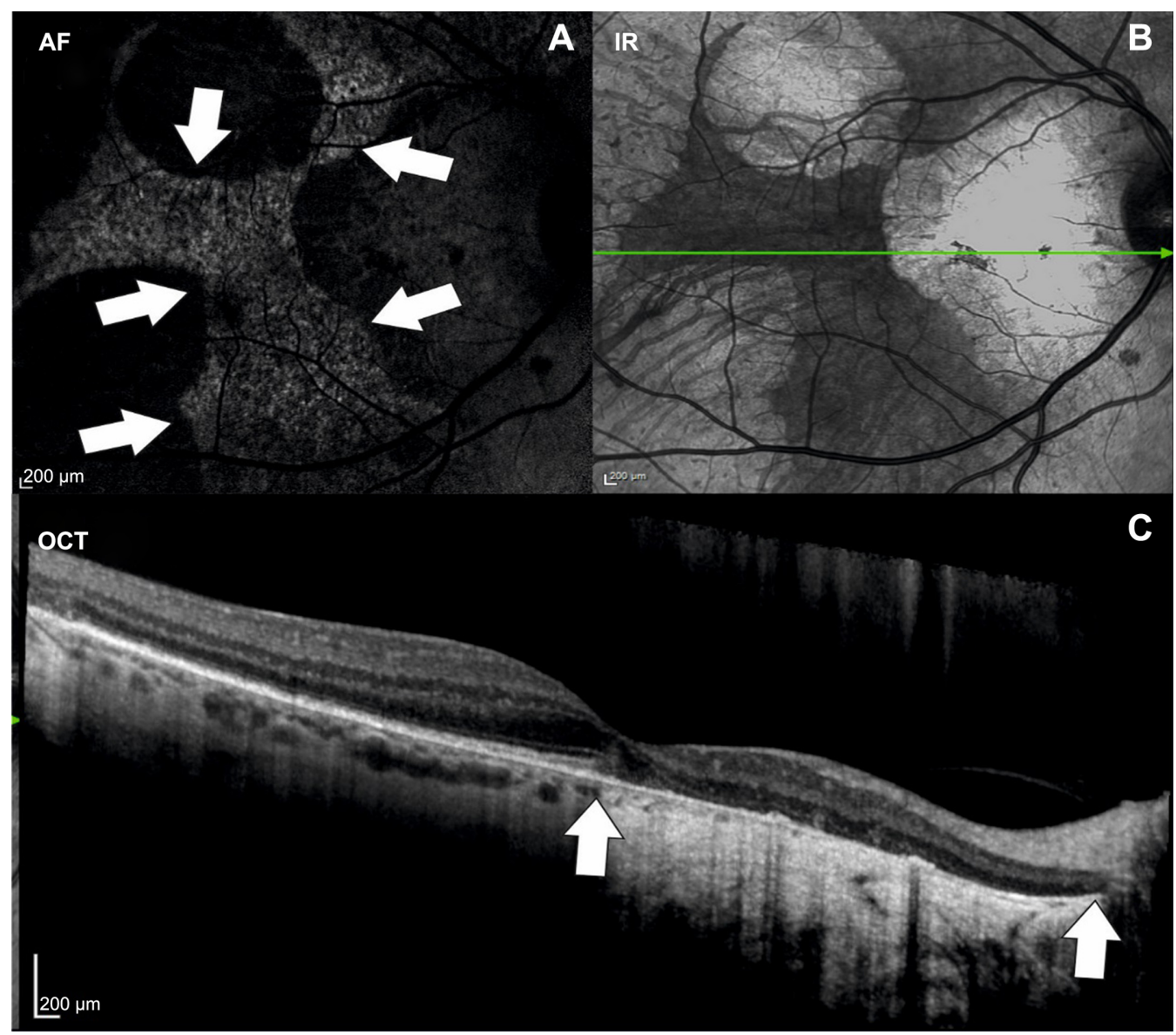

Figure 2 Multimodal imaging of a patient with choroideremia.

Notes: (A) Fundus AF showing an island of hyperfluorescent intact retina (delineated by white arrows). (B) IR image with corresponding OCT scan line (the green line represents the OCT scan direction) and (C) OCT images of the same patient. In OCT, the marked atrophy of the retinal pigment epithelium with homogenously increased reflectivity in OCT corresponds well with the borders seen in AF. There is a marked thinning of the outer nuclear layers and the choroidea is virtually absent, both hallmarks of choroideremia.

Abbreviations: AF, autofluorescence; OCT, optical coherence tomography; IR, infrared.

RPE65 gene is known to be expressed only in the RPE and being dominantly negative, we assume it causes RPE cell death; this phenotype provides an alternative pathway for the choroidal atrophy being RPE-driven in both diseases. Conversely, if CHM were a disease primarily of rod photoreceptor loss, then we might expect it to appear similar to the other forms of rod-cone dystrophy (retinitis pigmentosa).

Cone loss at the fovea and smaller atrophic-appearing cones at the edge of degeneration have also been found using adaptive optics scanning laser ophthalmoscopy. ${ }^{25}$ Functional tests such as mfERG and microperimetry are already employed to monitor therapeutic responses after gene replacement therapy. Future developments in imaging of retinal dystrophies should aim at identifying risk factors and reliable markers for disease progression. Here, several imaging modalities such as OCT angiography or fluorescence lifetime imaging ophthalmoscopy may provide disease specific information. In a recent report using en face OCT angiography, it has been shown that choriocapillaris nonperfusion was more pronounced than the retinal nonperfusion in patients with choroideremia. The observed choriocapillaris nonperfusion is thought to follow the RPE loss and may therefore possibly represent a marker for disease progression and/ or response to treatment. ${ }^{26}$ Fluorescence lifetime imaging ophthalmoscopy is a novel imaging modality able to quantify fluorescence lifetimes in the retina. ${ }^{27,28}$ In contrast to fundus autofluorescence, fluorescence lifetimes are influenced by metabolic changes within the retina. Therefore, this imaging modality may be valuable to identify areas at risk for disease progression in retinal dystrophies and may be a valuable tool to assess therapeutic responses. The exact sequel of structural changes occurring within the retina has received increasing interest in the last years in light of emerging therapies for retinal diseases.

\section{Genetics of choroideremia}

Choroideremia is caused by mutations in the CHM gene, located on the long arm of the $\mathrm{X}$ chromosome, encoding 
the $95 \mathrm{kDa}$ protein Rab escort protein 1 (REP1). The REP1 gene is ubiquitously expressed and acts as an escort protein of native regulators of intracellular trafficking. The deletions in the $\mathrm{CHM}$ gene causing choroideremia were first described in 1990. 29,30 Mutations in the CHM gene have been identified in European, ${ }^{31-35}$ Canadian, ${ }^{36}$ American, ${ }^{37}$ Japanese, ${ }^{38}$ and Chinese families. ${ }^{39}$ These mutations cause truncation or absence of REP1, which plays an essential role in the intracellular vesicular transport. REP1 is involved in lipid modification of Rab proteins by binding newly synthesized, unprenylated Rabs and presenting them to a catalytic Rab geranylgeranyltransferase or farnesyltransferase subunit for the geranylgeranyl transfer reaction. ${ }^{40}$ Furthermore, REP1 facilitates transport of prenylated Rabs to their specific destination membrane by binding to the hydrophobic prenylation motifs at their $\mathrm{C}$ termini. ${ }^{41} \mathrm{Rab}$ proteins are present in all cells and so far more than $50 \mathrm{Rab}$ proteins have been identified. ${ }^{42}$ Within the eye, many processes such as transport of proteins within the photoreceptors or regulation of phagocytosis and degradation of disc membranes shed from the outer segments of photoreceptors by RPE are reliant on Rab protein function. Generally, loss of REP1 is compensated by Rab escort protein 2, a protein that closely resembles REP1 and is encoded by the X-linked retrogene CHML (choroideremialike), which is located on chromosome $1 .{ }^{43}$ Most newly synthesized Rabs bind to one of the two REP proteins and therefore mutations in CHM generally do not lead to systemic disease. A possible explanation for the eye restricted phenotype is that some Rabs, such as Rab 27a which has a key role within the retina, ${ }^{44}$ are preferentially prenylated by REP1 or that REP2 prenylated Rab27a complexes may have a lower affinity to geranylgeranyltransferase. ${ }^{45}$ Therefore, the phenotype of choroideremia may be mainly caused by a tissue specific inability of REP2 to compensate for the function of REP1.

\section{Review of recent developments in therapies for choroideremia}

The retina is unusually accessible in the evaluation of trials of gene therapy. Not only can disease progression be imaged in nearly histological detail using high resolution OCT, but also there are several functional tests available to monitor therapeutic responses. Furthermore, modern vitreoretinal techniques allow for controlled and local delivery of gene replacement products to the affected tissue. Extensive preliminary work in mouse and dog models of retinal degeneration have shown that the use of adeno-associated vectors to deliver gene replacement therapy in the retina is safe and may result in stable and substantial restoration of the gene product and functional improvement. ${ }^{46}$ Of the many different adenoassociated virus (AAV) subtypes, AAV2 has a reasonable affinity for primate photoreceptors and RPE, ${ }^{47}$ but has the advantage over other serotypes in being well characterized in a number of animal models. Therefore, AAV2 has been used as the vector for gene replacement therapy in several gene therapy trials for retinal dystrophies. ${ }^{48-51}$ Because the amount of genetic material is limited by the packaging capacity of the vector, the size of gene to be replaced is of critical relevance. The limit for AAV vectors is estimated to be around $5 \mathrm{~kb}$ for single stranded DNA. ${ }^{52}$ With a total size of approximately $1.9 \mathrm{~kb}$, the coding sequence of REP1 fits well within the packaging limit of AAV2 with spare space for other regulatory elements, such as the promoter and poly-A signal sequences.

Preclinical studies have shown the feasibility of REP1 replacement therapy using AAV2 in $\mathrm{Chm}^{\text {null/+}}$ mice. In $\mathrm{Chm}^{\text {null/t+}}$ mice, selective ablation of $\mathrm{Chm}$ in photoreceptor cells or the RPE results in independent degeneration of both of these layers resembling the clinical phenotype of choroideremia. ${ }^{53}$ Using AAV2-REP1 delivered subretinally, a dose-dependent improvement of retinal function was observed in $\mathrm{Chm}^{\text {null/t+}}$ mice. These findings served as proof of concept for future clinical gene replacement therapies in humans. ${ }^{54}$

Retinal gene therapy in humans involves delivery of a viral vector to the photoreceptors and the RPE. In order to reach this tissue, a pars plana vitrectomy is necessary, followed by a small retinotomy and subretinal injection of fluid containing the vector. This procedure is highly complex because it must be done without damaging the fovea in a degenerate retina. The injection, to some extent, resembles subretinal injection of recombinant tissue plasminogen activator for the treatment and displacement of subretinal hemorrhage, although in these cases the retina is already detached and the visual acuity is severely compromised. In CHM, the potential risks of subretinal gene therapy should not be underestimated as the delivery of the vector entails detachment of the fovea which may lead to reduction of contrast sensitivity or even visual acuity. Furthermore, it is unknown whether or not the gene product or the vector might cause an immune reaction with potentially detrimental effects to the retina in some individuals.

Recently, preliminary results of a Phase I-II clinical trial assessing the effects of the AAV2-REP1 vector in six patients with choroideremia have been published. ${ }^{55}$ In this report, gene therapy with AAV2-REP1 was shown to be safe with a greater than 2- and 4-line improvement of visual acuity in two patients. Furthermore, it was shown that the five patients 
who received the full dose of AAV vector had improvements in mean retinal sensitivity. In this study, only patients with advanced disease with macular involvement were included. Four patients had near normal best corrected visual acuity at baseline and detachment of the fovea, which was required for gene replacement delivery, was shown to be well tolerated in these patients. Future prospects of gene replacement therapy in patients with choroideremia should target patients at an early disease stage when the retinal architecture and function are still intact. This has several implications for the clinician. Because there is now a potential therapy for patients with choroideremia and other retinal dystrophies, counseling of patients in clinics should include information on current results of gene replacement trials. Furthermore, genetic testing and molecular diagnosis of the DNA or messenger RNA causing retinal dystrophy should be used to support the clinical diagnosis. Even in the case of clinically confirmed choroideremia, genetic confirmation should be obtained in order to determine whether a patient is suitable for future gene replacement therapy trials. In addition, it is important to establish a database of patients with confirmed genetic mutations to inform patients of forthcoming gene replacement therapies and offer them the possibility of participating in future trials.

\section{Disclosure}

REM is a founder and director of NightstaRx Ltd, a choroideremia gene therapy company established by the University of Oxford and funded by the Wellcome Trust. MSZ reports no conflicts of interest in this work.

\section{References}

1. MacDonald IM, Sereda C, McTaggart K, Mah D. Choroideremia gene testing. Expert Rev Mol Diagn. 2004;4:478-484.

2. MacDonald IM, Mah DY, Ho YK, Lewis RA, Seabra MC. A practical diagnostic test for choroideremia. Ophthalmology. 1998;105:1637-1640.

3. Karna J. Choroideremia. A clinical and genetic study of 84 Finnish patients and 126 female carriers. Acta Ophthalmol Suppl. 1986;176:1-68.

4. Coussa RG, Kim J, Traboulsi EI. Choroideremia: effect of age on visual acuity in patients and female carriers. Ophthalmic Genet. 2012;33: 66-73.

5. Lyon MF. Gene action in the X-chromosome of the mouse (Mus musculus L.). Nature. 1961;190:372-373.

6. Godley BF, Tiffin PA, Evans K, Kelsell RE, Hunt DM, Bird AC. Clinical features of progressive bifocal chorioretinal atrophy: a retinal dystrophy linked to chromosome 6q. Ophthalmology. 1996;103:893-898.

7. Bowne SJ, Humphries MM, Sullivan LS, et al. A dominant mutation in RPE65 identified by whole-exome sequencing causes retinitis pigmentosa with choroidal involvement. Eur J Hum Genet. 2011;19:1074-1081.

8. Kmoch S, Majewski J, Ramamurthy V, et al. Mutations in PNPLA6 are linked to photoreceptor degeneration and various forms of childhood blindness. Nat Commun. 2015;6:5614.

9. McCulloch JC. The pathologic findings in two cases of choroideremia. Trans Am Acad Ophthalmol Otolaryngol. 1950;54:565-572.
10. Rafuse EV, McCulloch C. Choroideremia. A pathological report. Can J Ophthalmol. 1968;3:347-352.

11. MacDonald IM, Russell L, Chan CC. Choroideremia: new findings from ocular pathology and review of recent literature. Surv Ophthalmol. 2009;54:401-407.

12. Rodrigues MM, Ballintine EJ, Wiggert BN, Lee L, Fletcher RT, Chader GJ. Choroideremia: a clinical, electron microscopic, and biochemical report. Ophthalmology. 1984;91:873-883.

13. Joly S, Francke M, Ulbricht E, et al. Cooperative phagocytes: resident microglia and bone marrow immigrants remove dead photoreceptors in retinal lesions. Am J Pathol. 2009;174:2310-2323.

14. Sawa M, Tamaki Y, Klancnik JM Jr, Yannuzzi LA. Intraretinal foveal neovascularization in choroideremia. Retina. 2006;26:585-588.

15. Genead MA, Fishman GA. Cystic macular oedema on spectral-domain optical coherence tomography in choroideremia patients without cystic changes on fundus examination. Eye (Lond). 2011;25:84-90.

16. Cameron JD, Fine BS, Shapiro I. Histopathologic observations in choroideremia with emphasis on vascular changes of the uveal tract. Ophthalmology. 1987;94:187-196.

17. Goldberg NR, Greenberg JP, Laud K, Tsang S, Freund KB. Outer retinal tubulation in degenerative retinal disorders. Retina. 2013;33: 1871-1876.

18. Zinkernagel MS, Groppe M, MacLaren RE. Macular hole surgery in patients with end-stage choroideremia. Ophthalmology. 2013;120: $1592-1596$

19. Shinoda H, Koto T, Fujiki K, Murakami A, Tsubota K, Ozawa Y. Clinical findings in a choroideremia patient who underwent vitrectomy for retinal detachment associated with macular hole. Jpn J Ophthalmol. 2011;55:169-171.

20. Preising MN, Wegscheider E, Friedburg C, Poloschek CM, Wabbels BK, Lorenz B. Fundus autofluorescence in carriers of choroideremia and correlation with electrophysiologic and psychophysical data. Ophthalmology. 2009;116:1201-1209.e1-e2.

21. Edwards TL, Groppe M, Jolly JK, Downes SM, MacLaren RE. Correlation of retinal structure and function in choroideremia carriers. Ophthalmology. 2015;122:1274-1276.

22. Flannery JG, Bird AC, Farber DB, Weleber RG, Bok D. A histopathologic study of a choroideremia carrier. Invest Ophthalmol Vis Sci. 1990; 31:229-236.

23. Jacobson SG, Cideciyan AV, Sumaroka A, et al. Remodeling of the human retina in choroideremia: rab escort protein 1 (REP-1) mutations. Invest Ophthalmol Vis Sci. 2006;47:4113-4120.

24. Syed N, Smith JE, John SK, Seabra MC, Aguirre GD, Milam AH. Evaluation of retinal photoreceptors and pigment epithelium in a female carrier of choroideremia. Ophthalmology. 2001;108:711-720.

25. Syed R, Sundquist SM, Ratnam K, et al. High-resolution images of retinal structure in patients with choroideremia. Invest Ophthalmol Vis Sci. 2013;54:950-961.

26. Jia Y, Bailey ST, Hwang TS, et al. Quantitative optical coherence tomography angiography of vascular abnormalities in the living human eye. Proc Natl Acad Sci U S A. 2015;112:E2395-E2402.

27. Dysli C, Dysli M, Enzmann V, Wolf S, Zinkernagel MS. Fluorescence lifetime imaging of the ocular fundus in mice. Invest Ophthalmol Vis Sci. 2014;55:7206-7215.

28. Dysli C, Quellec G, Abegg M, et al. Quantitative analysis of fluorescence lifetime measurements of the macula using the fluorescence lifetime imaging ophthalmoscope in healthy subjects. Invest Ophthalmol Vis Sci. 2014;55:2106-2113.

29. Cremers FP, Sankila EM, Brunsmann F, et al. Deletions in patients with classical choroideremia vary in size from $45 \mathrm{~kb}$ to several megabases. Am J Hum Genet. 1990;47:622-628.

30. van de Pol TJ, Cremers FP, Brohet RM, Wieringa B, Ropers HH. Derivation of clones from the choroideremia locus by preparative field inversion gel electrophoresis. Nucleic Acids Res. 1990;18:725-731.

31. Beaufrere L, Tuffery S, Hamel C, et al. The protein truncation test (PTT) as a method of detection for choroideremia mutations. Exp Eye Res. 1997;65:849-854. 
32. Pascal O, Donnelly P, Fouanon C, Herbert O, Le Roux MG, Moisan JP. A new (old) deletion in the choroideremia gene. Hum Mol Genet. 1993; 2:1489.

33. Sankila EM, Tolvanen R, van den Hurk JA, Cremers FP, de la Chapelle A. Aberrant splicing of the CHM gene is a significant cause of choroideremia. Nat Genet. 1992;1:109-113.

34. Schwartz M, Rosenberg T, van den Hurk JA, van de Pol DJ, Cremers FP. Identification of mutations in Danish choroideremia families. Hum Mutat. 1993;2:43-47.

35. van Bokhoven H, Schwartz M, Andreasson S, et al. Mutation spectrum in the CHM gene of Danish and Swedish choroideremia patients. Hum Mol Genet. 1994;3:1047-1051.

36. Nesslinger N, Mitchell G, Strasberg P, MacDonald IM. Mutation analysis in Canadian families with choroideremia. Ophthalmic Genet. 1996;17:47-52.

37. Forsythe P, Maguire A, Fujita R, Moen C, Swaroop A, Bennett J. A carboxy-terminal truncation of 99 amino acids resulting from a novel mutation (Arg555 - > stop) in the CHM gene leads to choroideremia. Exp Eye Res. 1997;64:487-490

38. Fujiki K, Hotta Y, Hayakawa M, et al. REP-1 gene mutations in Japanese patients with choroideremia. Graefes Arch Clin Exp Ophthalmol. 1999; 237:735-740.

39. Zhou Q, Liu L, Xu F, et al. Genetic and phenotypic characteristics of three Mainland Chinese families with choroideremia. Mol Vis. 2012;18: 309-316.

40. Pereira-Leal JB, Hume AN, Seabra MC. Prenylation of Rab GTPases: molecular mechanisms and involvement in genetic disease. FEBS Lett. 2001;498:197-200.

41. Pylypenko O, Rak A, Reents R, et al. Structure of rab escort protein-1 in complex with rab geranylgeranyltransferase. Mol Cell. 2003;11: 483-494.

42. Pereira-Leal JB, Seabra MC. The mammalian Rab family of small GTPases: definition of family and subfamily sequence motifs suggests a mechanism for functional specificity in the Ras superfamily. $J$ Mol Biol. 2000;301:1077-1087.

43. Cremers FP, Armstrong SA, Seabra MC, Brown MS, Goldstein JL. REP-2, a Rab escort protein encoded by the choroideremia-like gene. J Biol Chem. 1994;269:2111-2117.

44. Tolmachova T, Anders R, Abrink M, et al. Independent degeneration of photoreceptors and retinal pigment epithelium in conditional knockout mouse models of choroideremia. J Clin Invest. 2006;116:386-394.
45. Larijani B, Hume AN, Tarafder AK, Seabra MC. Multiple factors contribute to inefficient prenylation of Rab27a in Rab prenylation diseases. J Biol Chem. 2003;278:46798-46804.

46. Acland GM, Aguirre GD, Bennett J, et al. Long-term restoration of rod and cone vision by single dose rAAV-mediated gene transfer to the retina in a canine model of childhood blindness. Mol Ther. 2005;12: 1072-1082.

47. Bennett J, Maguire AM, Cideciyan AV, et al. Stable transgene expression in rod photoreceptors after recombinant adeno-associated virus-mediated gene transfer to monkey retina. Proc Natl Acad Sci U S A. 1999;96: 9920-9925.

48. Maguire AM, High KA, Auricchio A, et al. Age-dependent effects of RPE65 gene therapy for Leber's congenital amaurosis: a phase 1 doseescalation trial. Lancet. 2009;374:1597-1605.

49. Jacobson SG, Cideciyan AV, Ratnakaram R, et al. Gene therapy for leber congenital amaurosis caused by RPE65 mutations: safety and efficacy in 15 children and adults followed up to 3 years. Arch Ophthalmol. 2012; 130:9-24.

50. Hauswirth WW, Aleman TS, Kaushal S, et al. Treatment of leber congenital amaurosis due to RPE65 mutations by ocular subretinal injection of adeno-associated virus gene vector: short-term results of a phase I trial. Hum Gene Ther. 2008;19:979-990.

51. Bainbridge JW, Mehat MS, Sundaram V, et al. Long-term effect of gene therapy on leber's congenital amaurosis. N Engl J Med. 2015;372(20): 1887-1897.

52. Wu Z, Yang H, Colosi P. Effect of genome size on AAV vector packaging. Mol Ther. 2010;18:80-86.

53. Tolmachova T, Wavre-Shapton ST, Barnard AR, MacLaren RE, Futter CE, Seabra MC. Retinal pigment epithelium defects accelerate photoreceptor degeneration in cell type-specific knockout mouse models of choroideremia. Invest Ophthalmol Vis Sci. 2010;51:4913-4920.

54. Tolmachova T, Tolmachov OE, Barnard AR, et al. Functional expression of rab escort protein 1 following AAV2-mediated gene delivery in the retina of choroideremia mice and human cells ex vivo. $J \mathrm{Mol} \mathrm{Med}$ (Berl). 2013;91:825-837.

55. MacLaren RE, Groppe M, Barnard AR, et al. Retinal gene therapy in patients with choroideremia: initial findings from a phase $1 / 2$ clinical trial. Lancet. 2014;383:1129-1137.
Clinical Ophthalmology

\section{Publish your work in this journal}

Clinical Ophthalmology is an international, peer-reviewed journal covering all subspecialties within ophthalmology. Key topics include: Optometry; Visual science; Pharmacology and drug therapy in eye diseases; Basic Sciences; Primary and Secondary eye care; Patient Safety and Quality of Care Improvements. This journal is indexed on Submit your manuscript here: http://www.dovepress.com/clinical-ophthalmology-journal

\section{Dovepress}

PubMed Central and CAS, and is the official journal of The Society of Clinical Ophthalmology (SCO). The manuscript management system is completely online and includes a very quick and fair peer-review system, which is all easy to use. Visit http://www.dovepress.com/ testimonials.php to read real quotes from published authors. 